\title{
Charge-state evolution of highly charged ions transmitted through microcapillaries
}

\author{
K. Tökési, ${ }^{*}$ L. Wirtz, C. Lemell, and J. Burgdörfer \\ Institute for Theoretical Physics, Vienna University of Technology, Wiedner Hauptstraße 8-10, A-1040 Vienna, Austria
}

(Received 18 May 1999; published 18 January 2000)

\begin{abstract}
The charge-state evolution of highly charged ions transmitted through microcapillaries is studied theoretically by a classical trajectory Monte Carlo simulation. The interaction of highly charged ions with the internal surface of the capillary is treated within the framework of dielectric response theory. We analyze the distance of closest approach and the angular distributions of the highly charged ions at the exit of the microcapillary. We find the charge-state fraction of transmitted $\mathrm{N}^{6+}$ projectiles, in good agreement with first measurements. Moreover, our calculations indicate that grazing collisions with the microcapillary surface hold the promise of direct observation of charge transfer and hollow-atom formation at a large distance from the surface.
\end{abstract}

PACS number(s): 34.50.Dy

Collisions between highly charged ions (HCI) and solid surfaces are currently at the center of numerous experiments and theoretical investigations [1-8]. The main motivation of these efforts is the study of the fundamental interaction mechanism between the HCI and the solid surfaces. An accurate knowledge of these processes is very important for the use of HCI as a surface diagnostic tool as well as for surface modifications (see, e.g., [9]).

From a number of experimental and theoretical studies the following scenario of the HCI-surface interaction has emerged: When a highly charged ion approaches a solid surface, one or more electrons can be resonantly captured at a characteristic distance $\left(d_{c}\right)$ into Rydberg states of the projectile with large principal quantum numbers $n_{c}$. As a result, a multiply excited Rydberg atom with inner-shell vacancies, a so-called hollow atom, is created. For metal surfaces, charge transfer of the weakly bound conduction band electrons into a highly charged ion sets in at large distances from the surface. These hollow atoms are often referred to as abovesurface hollow atoms or hollow atoms of the first generation (HA1). The classical over-the-barrier (COB) model [2] is widely used to estimate this distance where the first resonant charge transfer can take place in the electronic shells of the projectile with principal quantum number of the order $n_{c}$. The COB model also gives information about the interaction time for a HCI above the target surface and the neutralization dynamics. The interaction time available between first capture and impact on the surface is limited by the image acceleration and is typically of the order of $t_{I}<10^{-13} \mathrm{sec}$. During this time interval complete relaxation to the neutral ground state is impossible. Therefore, the ion reaches the surface in a multiply excited state. At or below the surface the outer charge cloud is replaced by a much tighter charge cloud with a radius of the order of the bulk value of the metallic screening radius, forming a hollow atom of the second generation (HA2). Therefore, close collision at the surface and bulk penetration provide a much more efficient pathway to complete relaxation. In turn, however, the information on the

\footnotetext{
*Permanent address: Institute of Nuclear Research of the Hungarian Academy of Sciences, (ATOMKI), H-4001 Debrecen, P.O. Box 51, Hungary.
}

above-surface hollow atom is (to a large extent) erased, as its direct observation is limited to the time interval $t_{I}$. In particular, many properties of hollow atoms such as long-lived multiply excited resonances escape the observation.

Very recently, interactions of HCI with internal surfaces in microcapillaries have been introduced as an alternative technique to study above-surface processes [10,11]. Ions traveling approximately parallel to the capillary axis will be attracted by image forces toward the cylindrically shaped internal wall of the capillary (Fig. 1). While the dominant fraction of projectiles will either exit without undergoing charge transfers (trajectory type 1, Fig. 1) or undergo close collisions with the surface similar to conventional grazing surface collisions (trajectory type 2), trajectories of type 3 will undergo only large-distance "above-surface" collisions near the exit surface of the wall. These ions can escape prior to hitting the wall in close collisions with the capillary and, hence, preserve the memory of the above-surface hollowatom formation.

In this Rapid Communication, transmission of HCI through microcapillaries is studied by a classical trajectory Monte Carlo method. The interaction between the HCI and the capillary surface is taken into account by the image interaction within the framework of dielectric response. For later comparison with the experiment of Ninomiya et al. [11] we perform the simulations for a metallic microcapillary of Ni. As projectile we use $\mathrm{N}^{6+}$ with an energy of $2.1 \mathrm{keV} / \mathrm{amu}$. Furthermore, we assume that the isotropic solid located outside the cylinder of radius $\rho_{0}$ can be characterized by a dielectric response function $\epsilon(k, \omega)$ that is a function of the

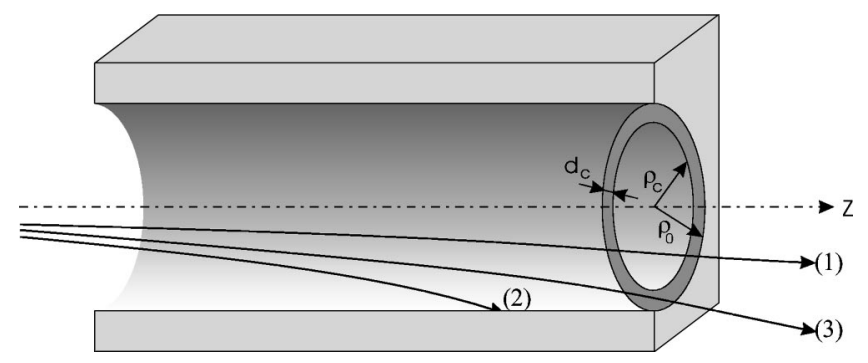

FIG. 1. Sketch of the ideal microcapillary with typical ion trajectories. $\rho_{c}$ is the critical capture radius and $d_{c}$ is the critical capture distance. 
frequency $\omega$ and the wave number $k$ of the electromagnetic disturbance. Following $[12,13]$ we determine $\varepsilon(k, \omega)$ in terms of a sum of Drude-type functions and fit the limit $\varepsilon(k=0, \omega)$ to the optical data for $\mathrm{Ni}$ as compiled by Edward [14]. The extension to finite $k$ values proceeds by employing a dispersion relation $\omega(k)$ that interpolates between the plasmon and the quasifree particle limits. The resulting $\varepsilon(k, \omega)$ satisfies the generalized Thomas-Reiche-Kuhn sum-rules [15] for response functions. The boundary-value problem determining the interaction potential between the charge $q$ and the capillary wall can be conveniently solved using cylindrical coordinates $[16,17]$. The charge (e.g., of the ion) is located at $\vec{v} t=\vec{r}_{1}=\left(\rho_{1}, \phi_{1}, z_{1}\right)$, where $\vec{v}$ is the velocity of the ion.

In our simulations a Ni capillary with a nominal radius $\rho_{0}$ of $125 \mathrm{~nm}$ ( $\approx 2360$ a.u. $)$ and length $L=1.5 \mu \mathrm{m} \approx 28400$ a.u. was used. Since ensembles of experimentally available capillaries do not form ideal cylinders but have "bumps," bottlenecks, and inhomogeneities, these deviations from the ideal geometry are taken into account within a statistical ensemble. The realistic capillary is assumed to form terraces with lengths randomly distributed between 20 and $50 \mathrm{~nm}$ ( $\approx 400$ to 1000 a.u.). The terrace height distribution is simulated by allowing the cylindrical radius to vary randomly by $\pm 5 \%$ around its nominal value. Furthermore, we take fringe-field effects due to the edge at the capillary end into account. The potential for the infinitely extended cylinder is replaced near the exit plane by a three-dimensional wedge potential [18]. For estimating corrections due to fringe fields, the cylindrically shaped surface is replaced by a planar surface (i.e., a Cartesian wedge). This is justified since the relevant ensemble of trajectories we analyze in the following is near the exit surface close to within $\approx 20$ a.u. of one of the walls (forming the wedge), while the opposite wall is at that point about $250 \mathrm{~nm}(\approx 4700$ a.u. $)$ apart. Note, however, that such an approximation does not hold in the interior of the capillary where the distances to the opposing walls can be comparably large.

As soon as the ion approaches the wall within a distance $d_{c}$ for capture, the electronic and ionic dynamics have to be treated self-consistently, as charge transfer influences the ionic trajectory. The electronic dynamics is simulated within a Monte Carlo approach: electron capture, resonant ionization, radiative decay and nonradiative decay are followed as a stochastic event-by-event sequence with rates taken from the COB model for capture and loss [2] modified for the cylindrical geometry. Within the framework of the independent-particle model, multiple-electron transfer is included. Auger and radiative rates are taken from atomic structure codes $[19,20]$. Note that for any event the influence of previous events along the history of one trajectory (ionic position, energy gain, charge state, shell occupation) is taken into account through variation of relevant parameters that determine the probability for subsequent events. Details of simulations will be given elsewhere [21].

The influence of the charge-transfer process on trajectories passing by the exit surface is illustrated in Fig. 2, where we compare trajectories with frozen charge states as used in

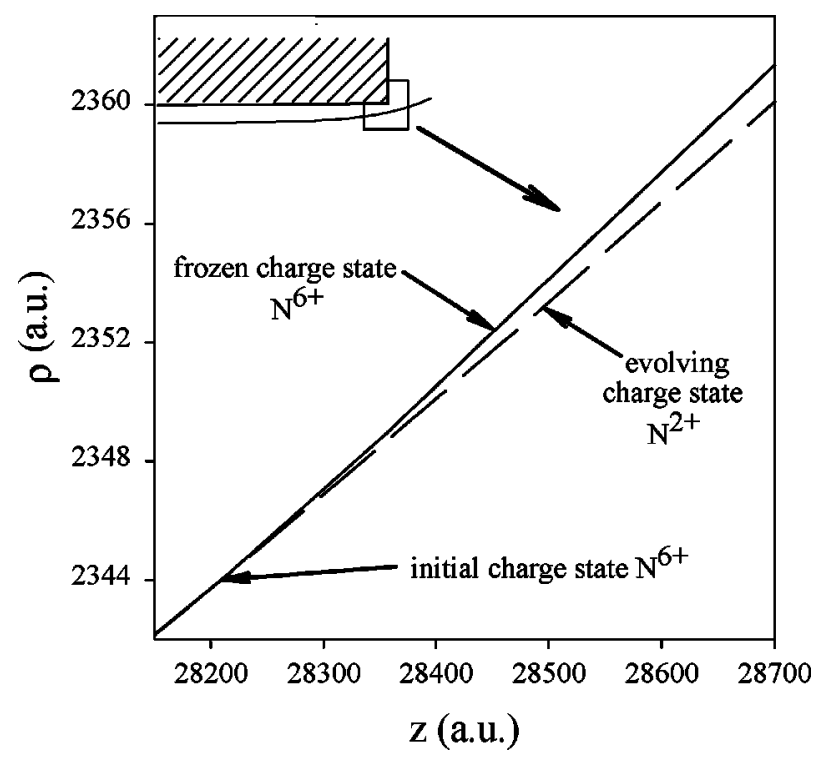

FIG. 2. Comparison between ionic trajectories of incident $\mathrm{N}^{6+}$ with frozen charge state $q=6$ and dynamically evolving charge state reaching $q=2$.

a preliminary investigation [22] with a self-consistent solution. For the same incident charge state, the scattering angle for the frozen charge state is generally larger than for the case of dynamically evolving projectile charges due to the larger image acceleration in the absence of the neutralization process. For the same reason, the dynamical evolution of the charge state reduces the number of close fly-by events and increases the average distance of closest approach to the exit edge.

In order to study the hollow-ion formation in microcapillary transmission, we have performed a simulation with an ensemble of $5 \times 10^{7}$ primary trajectories. The spatial distribution of the ensemble is uniform across the opening of the capillary cylinder. As a first test case we analyze the charge state distribution of outgoing $\mathrm{N}^{q+}$ ions for which experimental data are available [11]. To simulate the spread of the incident beam, we use a Gaussian angular distribution of $\theta_{i}$ with a full width at half-maximum (FWHM) of $2^{\circ}$, which is the estimated experimental value [23]. Extensions to more highly charged ions are straightforward and yield qualitatively similar results. It is important to realize that the charge-state distribution of the ensemble reaches its asymptotic stable limit only after the ion is about $100 \mu \mathrm{m}$, i.e., almost macroscopic distances, downstream from the exit surface when the Auger relaxation is complete. This is in sharp contrast to conventional specular reflection surface scattering processes, where, as a result of close collisions, the final charge state is reached on a receding branch of the trajectory within a few angstroms above the surface. The long relaxation time toward the asymptotic charge-state distribution $(\sim 0.1 \mathrm{nsec})$ is due to a sequence of comparatively slow Auger processes involving asymmetric configurations $\left(n l, n^{\prime} l^{\prime}\right)$ with $n \gg n^{\prime}$. The overall agreement with the data is remarkably good (Fig. 3). The point to be noted is that only about $15 \%$ of the transmission through the capillary, while the remainder hits the entrance surface, undergoes close col- 


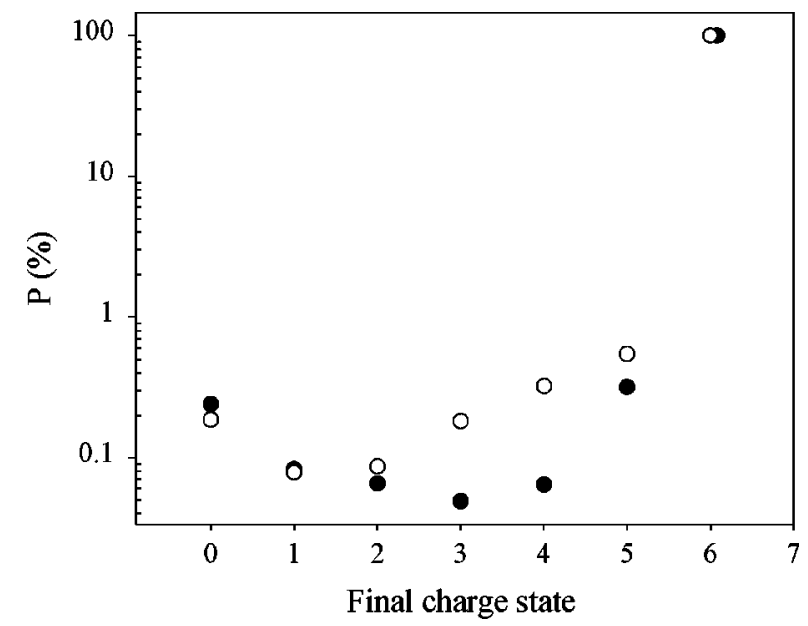

FIG. 3. Final charge-state distribution of incident $2.1 \mathrm{keV} / \mathrm{amu}$ $\mathrm{N}^{6+}$ ions transmitted through the Ni microcapillary. Open circles, experiment [11]; solid circles, simulation.

lisions with the walls, and is stopped in the capillary material. Within the subensemble of exiting ions, the incident charge state strongly dominates $(\sim 99 \%)$. The ratio of the incident charge-state fraction to all other charge states is directly related to the ratio of the entire capillary cross section of the cylinder to the area subtended by a ring of thickness $d_{c}$ (Fig. 1), and therefore provides a direct test for the distance of first capture. The present simulation gives overall good agreement for all ionic charge states on an absolute scale. The fairly flat and uniform distribution over all other charge states $(q=0,1,2,3,4,5)$ is a unique feature of distant collisions with the internal microcapillary surface, not previously observable in ion-atom, ion-solid, and grazing ionsurface collisions. It should be noted that the only fraction of neutrals $(q=0)$ in the experiment may contain a sizable contribution from projectiles undergoing close collisions near the exit surface and exit completely relaxed in the neutral ground state. As the final charge-state distribution depends also on a multitude of Auger relaxation processes, the distribution of electrons among $n$ shells of the hollow atom also appears to be predicted reasonably well. There are, however, a few noticeable discrepancies for charge states $q=3$ and 4 that correspond to electron configurations with a net capture of two or three electrons, respectively. Their apparent enhanced stability could possibly be due to the presence of extremely long-lived metastable configurations that are currently not yet included in the simulation. Possible candidates for such metastable multiply excited states could include high-spin states or high angular-momentum states [24] or, alternatively, planetary type configurations [25]. This aspect will be explored in the future in more detail.

From our simulations we can also extract the angular $(\theta)$ distribution as well as the distance of closest approach $(b)$ distribution relative to the nearest exit edge (Fig. 4). To the extent that the $\theta$ and $b$ distributions are correlated, measurements of the charge-state distributions in coincidence with the scattering angle would provide the means to resolve subsequent stages of the hollow-atom formation above the surface. To demonstrate the angular separation, the angular dis-

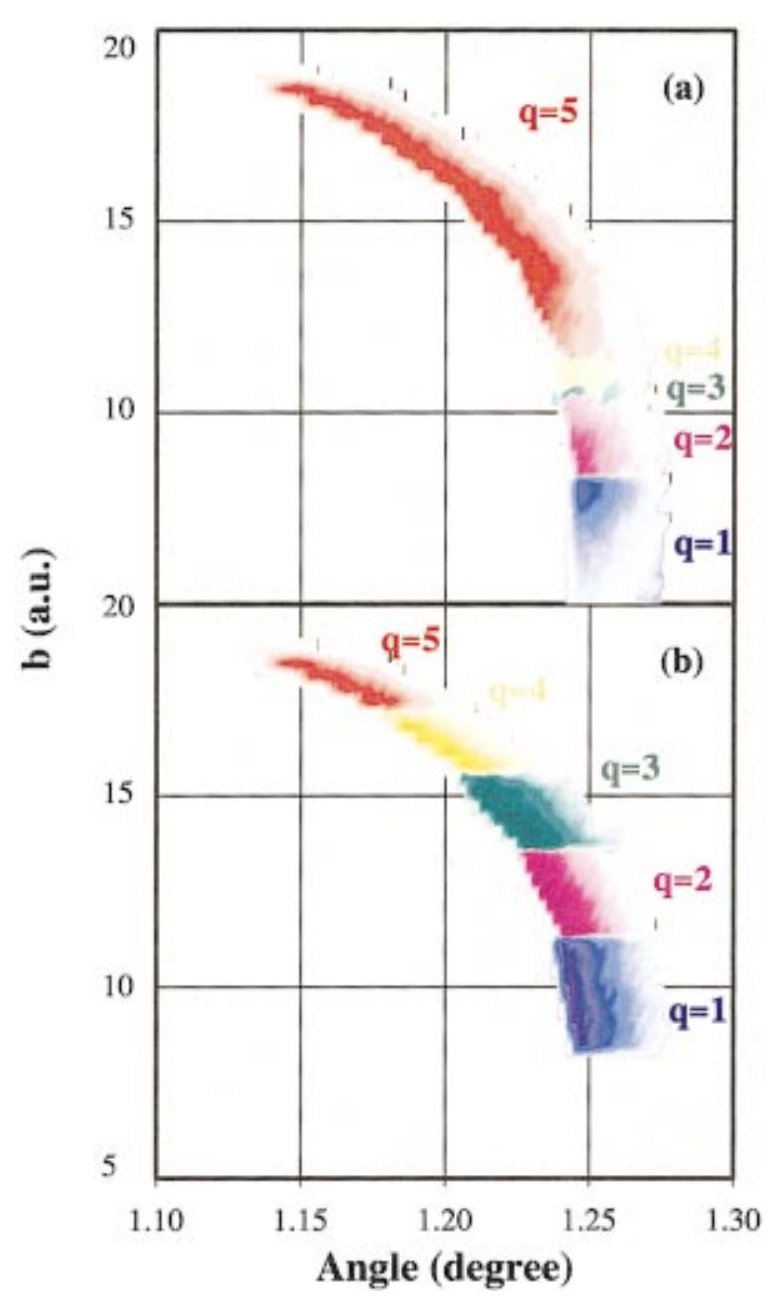

FIG. 4. (Color) Two-dimensional $P^{q+}(\theta, b)$ distribution of scattering angle $\theta$ and distance of closest approach $b$. (a) Contour plot for asymptotic charge-state distributions $q_{\text {asymp }}$; (b) contour plot of the distribution at a distance of $d_{c}\left(q \neq q_{\text {asymp }}\right)$.

tribution of the incident beam has been restricted to a FWHM of $0.2^{\circ}$. Note that for higher incident charge states the angular separation can be clearly seen, even with much less stringent requirements on the initial beam divergence. Figure 4 displays the two-dimensional distributions $P^{q+}(\theta, b)$ for $q=1, \ldots, 5$, where panel (a) represents the asymptotic observable distributions, while (b) gives the distribution in close proximity to the exit surface. We omit the $q=0$ component because of the possible contamination by contributions from "hard" collisions. Similar to grazingincidence surface collisions [3,26], the image acceleration of the HCI toward the wall manifests itself as a pronounced shift in the angular distribution towards larger scattering angles. We indeed observe a banana-shaped $\theta-b$ correlation pattern with a very well localized angular distribution. It is noteworthy that the angular distribution for each charge state is much narrower $\left(\mathrm{FWHM} \leqslant 0.05^{\circ}\right.$ ) than the initial angular divergence of the beam $\left(\mathrm{FWHM}=0.2^{\circ}\right)$. This is a direct consequence of the intrinsic high selectivity among all initial conditions that lead to transmission through the microcapillary accompanied by charge exchange of a given charge state. Low charge states are strongly correlated with closer 
fly-by's (small $b$ 's) and larger scattering angles $\left(\theta \simeq 1.25^{\circ}\right)$, while the effective single capture channel $(q=5)$ extends from the threshold angle $\theta_{c}=1.13^{\circ}$ for single capture to larger angles. Notice that below $\theta_{c}$ no capture takes place, since such angles would correspond to $b>d_{c}$. The comparatively broad angular distribution for the charge state $q=5$ is not due to a wider angular range for single capture in distant fly-by events, but is due to the Auger decay of hollow atoms of (initially) lower charge states. To illustrate this effect we show in Fig. 4(b) the $\theta-b$ correlation pattern for the same charge states but during flight as the ions pass through the exit surface. The evolution of this distribution to the one in Fig. 4(a) is exclusively due to the Auger cascade, while the angular and distance of closest approach distribution pattern is already essentially frozen out. This simulation suggests that different stages of the hollow-atom (or ion) formation should be directly accessible in future experiments, provided that an angular resolution of the order of $\theta \leqslant 0.05^{\circ}$ can be achieved. Usage of higher charge states would greatly facilitate the observation of the correlation pattern. Even more detailed information could be extracted when the decay of the multiply excited state by photon or electron emission could be measured in coincidence with the scattering angle.

In summary, we have presented Monte Carlo simulations of the transmission of multiply charged ions passing through a microcapillary target. The simulation treats the ionic and electronic degrees of freedom simultaneously. We find the fraction of transmitted projectiles that are partially neutralized to be in good agreement with recent experiments. Moreover, the charge-state-dependent correlation between the scattering angle and distance of closest approach predicts that angular-resolved charge-state distributions may provide direct information on the evolution of the charge cloud of a hollow atom at large distances from the surface.

This work has been supported by the Austrian Fonds zur Förderung der wissenschaftlichen Forschung (P12470-TPH).
[1] J.P. Briand et al., Phys. Rev. Lett. 65, 159 (1990).

[2] J. Burgdörfer, P. Lerner, and F.W. Meyer, Phys. Rev. A 44, 5647 (1991); J. Burgdörfer, in Fundamental Processes and Applications of Atoms and Ions, edited by C.D. Lin (World Scientific, Singapore, 1993), pp. 517-614.

[3] H. Winter, Europhys. Lett. 18, 207 (1992).

[4] F.W. Meyer et al., Phys. Rev. A 48, 4479 (1993).

[5] J. Das and R. Morgenstern, Comments At. Mol. Phys. 29, 205 (1993).

[6] N. Stolterfoht et al., Phys. Rev. A 52, 445 (1995).

[7] C. Lemell et al., Phys. Rev. A 53, 880 (1996).

[8] A. Arnau et al., Surf. Sci. Rep. 27, 113 (1997).

[9] See, for example, Proceedings of the 8th International Conference on the Physics of Highly Charged Ions (1996) [Phys. Scr. T73 (1997)]; Proceedings of the 9th International Conference on the Physics of Highly Charged Ions (1998) [ibid. T80 (1999)].

[10] Y. Yamazaki et al., J. Phys. Soc. Jpn. 65, 1192 (1996).

[11] S. Ninomiya et al., Phys. Rev. Lett. 78, 4557 (1997).

[12] J.C. Ashley et al., Thin Solid Films 60, 361 (1979).

[13] B. Gervais et al., Phys. Rev. A 53, 3189 (1996).

[14] D.W. Lynch and W.R. Hunter, in Handbook of Optical Constant of Solids, edited by D.P. Edward (Academic Press, Lon- don, 1985).

[15] P. Nozieres and D. Pines, The Theory of Quantum Liquids (Addision-Wesley, Reading, MA, 1990).

[16] D. Chan and P. Richmond, Surf. Sci. 39, 437 (1973).

[17] J.D. Jackson, Classical Electrodynamics, 2nd ed. (Wiley, New York, 1975).

[18] W.R. Smythe, Static and Dynamic Electricity, 2nd ed. (McGraw-Hill, New York, 1950).

[19] J. Limburg et al., Phys. Rev. A 51, 3873 (1995); M. Grether et al., ibid. 52, 426 (1995); N. Stolterfoht et al., ibid. 52, 445 (1995); N. Vaeck and J.E. Hansen, J. Phys. B 28, 3523 (1995).

[20] E.J. McGuire, Phys. Rev. A 3, 587 (1971); D.L. Walters and P. Bhalla, ibid. 3, 1919 (1971); C.P. Bhalla, and M. Hein, Phys. Rev. Lett. 30, 39 (1973); R. Díez Muiño et al., Phys. Rev. A 57, 1126 (1998).

[21] K. Tökési et al., (unpublished).

[22] K. Tökési et al., Nucl. Instrum. Methods Phys. Res. B 154, 307 (1999)

[23] Y. Yamazaki, (private communications).

[24] Y. Yamazaki (unpublished).

[25] K. Richter and D. Wintgen, J. Phys. B 23, L197 (1990).

[26] J. Burgdörfer, and F. Meyer, Phys. Rev. A 47, 20 (1993). 